\title{
Rational design of mimetic peptides based on aldo-ketoreductase enzyme as asymmetric organocatalysts in aldol reactions
}

\begin{abstract}
Peptides as a kind of important chiral scaffold are broadly identified for their obvious advantages, diverse structures and accessibility. Based on promiscuous aldo-keto-reductase enzymes, several mimetic peptides were designed which were synthesized and tested as multifunctional organocatalysts in direct asymmetric aldol reactions. The corresponding aldol products were produced with high yields (up to 97\%) and excellent diastereoselectivities (up to 99/1) and enantioselectivities (>98\%) under mild reaction selectivity and enantioselectivity. The secondary structures of peptide catalysts provide an understanding of their mechanism.
\end{abstract}

Keyword: Rational design; Mimetic peptides; Aldo-ketoreductase enzyme; Asymmetric organocatalysts; Aldol reactions 